\title{
ELABORACIÓN Y VALIDACIÓN DE INSTRUMENTO PARA MEDIR PRÁCTICAS SUSTENTABLES QUE CREAN VALOR EN ORGANIZACIONES AGRÍCOLAS
}

\author{
CONSTRUCTION AND VALIDATION OF A QUESTIONNAIRE \\ TO MEASURE SUSTAINABLE PRACTICES THAT CREATE VALUE \\ IN AGRICULTURAL ORGANIZATIONS
}

\begin{abstract}
${ }^{1}$ Rosa Elena De Anda-Montaño, ${ }^{2}$ Raúl Portillo-Molina, 3Myrna Delfina López-Noriega, ${ }^{4}$ María de los Ángeles Cervantes-Rosas
\end{abstract}

${ }^{1}$ Estudiante del Doctorado en Sustentabilidad, incorporado al PNPC del Conacyt, de la Universidad Autónoma de Occidente, Unidad Regional Guasave, Sinaloa, México.

${ }^{2}$ Doctor en Estudios fiscales por la Universidad Autónoma de Sinaloa, incorporado al PNPC del Conacyt. PTC investigador de la Universidad Autónoma de Occidente, Unidad Regional Guasave, Sinaloa, México.

${ }^{3}$ Doctora en Administración por la Universidad del Sur. Investigadora de la Universidad Autónoma del Carmen, Ciudad del Carmen, Campeche, México.

${ }^{4}$ Doctora en Administración por la Universidad Autónoma de Occidente, incorporado al PNPC del Conacyt. PTC Investigador de la Universidad Autónoma de Occidente, Unidad Regional Guasave, Sinaloa, México

\author{
${ }^{1}$ rosa.deanda@udo.mx; ${ }^{2}$ raul.portillo@udo.mx; \\ ${ }^{3}$ mdlopez@pampano.unacar.mx; ${ }^{4}$ maría.cervantes@udo.mx
}

\section{RESUMEN}

Contextualización: Guasave es un municipio ubicado en el noroeste de México, forma parte de las 18 municipalidades del Estado de Sinaloa, es considerada como una de las regiones más ricas en la actividad agrícola del país, de acuerdo con el volumen de producción y superficie sembrada a nivel nacional.

Vacío de conocimiento: el calentamiento global, la contaminación y erosión de suelos y aguas, han afectado gravemente a la agricultura; por ello, es necesario que se instrumenten estrategias sustentables que permitan a este sector seguir desarrollándose. La importancia de este artículo recae en hacerle frente a la escasez de instrumentos que coadyuven a la creación de valor en empresas dedicadas a esta actividad, la implementación de éste permitirá obtener información empírica útil para la identificación de las prácticas sustentables que generan valor a las organizaciones agrícolas. 
Propósito del estudio: el objetivo de este artículo es dar a conocer el proceso de construcción y validación, así como divulgar el instrumento "Prácticas sustentables para la creación de valor en organizaciones agrícolas" del norte de Sinaloa, México.

Metodología: son 38 ítems de escala valorativa, organizados en las tres dimensiones de desarrollo sustentable y creación de valor los que integran el instrumento. El enfoque retomado fue el cuantitativo, la población objetivo fue de 32 organizaciones agrícolas del municipio para la prueba de fiabilidad mediante el coeficiente de consistencia interna Alfa de Cronbach y 10 expertos para la validación.

Resultados y conclusiones: los resultados obtenidos permiten afirmar que el instrumento es apropiado para medir las prácticas sustentables que crean valor en las organizaciones agrícolas de la parte norte de la entidad, al determinarse un coeficiente de 0.941 y una pertinente validez.

Palabras Claves: creación de valor; fiabilidad; agricultura; sustentabilidad y validez.

\section{ABSTRACT}

Contextualization: Guasave is a municipality located in the northwest of Mexico, it is part of the 18 municipalities of the State of Sinaloa, it is considered one of the richest regions in the country's agricultural activity, according to the volume of production and nationally sown area.

Knowledge gap: The global warming, the pollution and erosion of soils and waters have seriously affected agriculture; therefore, it is necessary to implement sustainable strategies that allow this sector to continue developing. The importance of this article lies in addressing the shortage of instruments that contribute to the creation of value in companies dedicated to this activity. The implementation of this will allow us to obtain useful empirical information for the identification of sustainable practices that generate value for agricultural organizations.

Purpose: The purpose of this article is to publicize the construction and validation process, as well as to disseminate the instrument "Sustainable practices for value creation in agricultural organizations" in northern Sinaloa, Mexico.

Methodology: There are 38 items of value scale, organized in the three dimensions of sustainable development and value creation that make up the instrument. The approach used was quantitative, the target population was 32 agricultural organizations in the municipality for the reliability test using Cronbach's Alpha internal consistency coefficient and 10 experts for validation.

Results and conclusions: The results obtained allow us to affirm that the instrument is appropriate to measure the sustainable practices that create value in the agricultural organizations of the northern part of the entity, when determining a coefficient of .941 and a pertinent validity.

Keywords: creation of value; reliability; agriculture; sustainability and validity. 


\section{RESUMEN GRÁFICO}

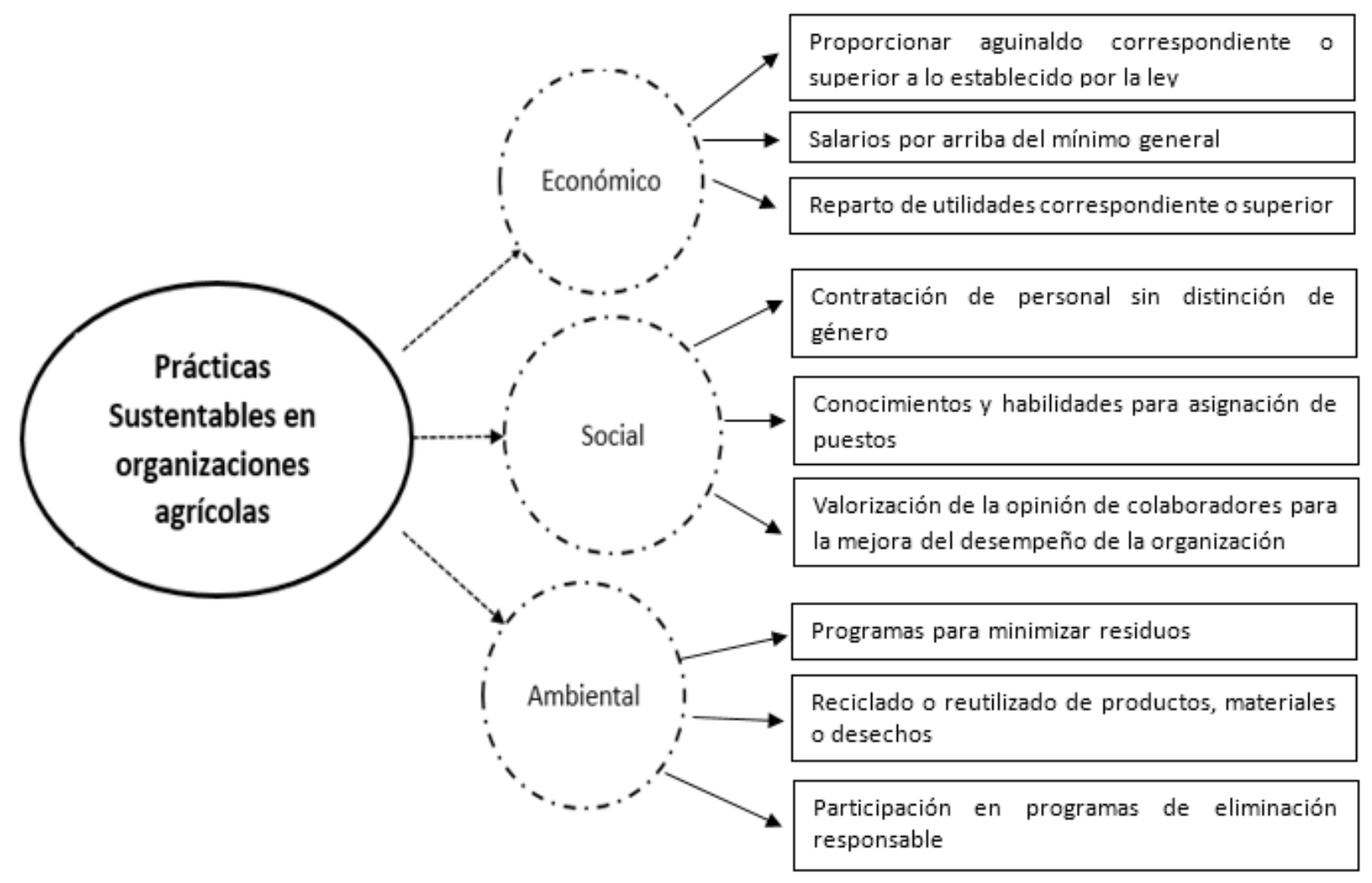

Figura 1. Principales prácticas sustentables que generan valor a las organizaciones agrícolas.

Fuente: Autores

\section{INTRODUCCIÓN}

Las prácticas sustentables en organizaciones agrícolas son una alternativa para que dichas asociaciones basen su quehacer en los ejes económico, social y ambiental para el cumplimiento de los 17 objetivos del desarrollo sustentable (Carro, Reyes, Rosano, Garnica y Pérez, 2017; Čater, et al., 2009; Jane, 2013, Rodríguez y Ricart 2010; Paternoster, 2011; Leal, 2009) y con ello contribuir a garantizar el futuro de la humanidad (Bell \& Morse, 2018); retribuyendo a la sociedad el cuidado del entorno por permitirles desarrollar su actividad y generando beneficios económicos; garantizando la permanencia en el mercado, generando valor a sus intangibles a través de su imagen y reputación (Carmona y
Magán, 2008; Orsato, 2006; Rodríguez y Ricart, 2000; Carro et al. 2017; Carrillo, 2011; Hart y Milstein, 2003 y Zulueta et al., 2013).

En ese sentido, la propuesta de triple línea de balance (término concerniente a los negocios sostenibles que hace referencia al impacto que la actividad de una empresa tiene en las tres dimensiones: económica, social y ambiental (Henriques y Richardson, 2013) señala las directrices en las cuales tienen que desempeñar sus actividades cualquier empresa que pretenda laborar bajo el Desarrollo Sustentable (DS) (Escobedo y García, 2018), dando lugar al concepto de Sustentabilidad Corporativa (SC) (Elkington, 1994). 
En este sentido, y con base en lo expuesto, las prácticas sustentables son una herramienta fundamental para el logro de la SC. Como resultado de esta indagación, las principales acciones llevadas a cabo por las organizaciones agrícolas del norte de Sinaloa son: proporcionar salarios, aguinaldos y reparto de utilidades por arriba de lo establecido por la ley (eje económico); contratar personas sin hacer distinciones de género, priorizar conocimientos y habilidades para asignar puestos y valorar la opinión de sus colaboradores para mejora de la organización (eje social), y por último, tener programas para minimizar residuos, así como participación en programas responsables de eliminación de envases de agroquímicos y reutilizar o reciclar productos o materiales (eje ambiental).

Ahora bien, al hablar de la actividad principal de estas organizaciones, la agricultura es fundamental para los países al garantizar la seguridad alimentaria, es fuente de ingresos y empleos, además de formar parte de cadenas agroalimentarias de producción (Molina y Victorero, 2015; Corona, 2016; IAEA, 2012). En México, esta actividad es significativa dentro de las actividades primarias, aportando en gran medida al desarrollo del país: solamente en el 2015, aportó aproximadamente un 63 por ciento del total aportado al Producto Interno Bruto (PIB) por ese sector primario, fruto de sus más de 27 millones de hectáreas que operan como superficie agrícola, generando casi ocho millones de empleos (Corona, 2016; SAGARPA, 2016).

De esos datos estadísticos, el Estado de Sinaloa destaca por ser es una de las entidades con mayor producción agrícola del país, al destinar 1,2 millones de hectáreas a esta actividad, produciendo más de 12 millones de toneladas de alimentos (CODESIN, 2017). Dentro del Estado, en el municipio de Guasave, esta actividad es la principal fuente de ingresos y empleos, generando en el año 2015 un valor de producción de cerca de 3.691 millones de pesos mexicanos (SIAP, 2018).

En relación a la problemática de esta actividad, distintas investigaciones han comprobado que el rendimiento de la producción ha disminuido debido a que la superficie está cada vez más erosionada y contaminada por el uso indiscriminado de productos químicos, tales como fertilizantes, plaguicidas, pesticidas, entre otros (Jaramillo et al., 2007; Sinergia, 2009); además el uso desmedido de productos químicos ha generado graves alteraciones tanto en ecosistemas como en organismos vivos (Martínez et al. 2016).

Es por ello, que las organizaciones agrícolas se han visto en la necesidad de buscar alternativas que disminuyan estos daños, todo esto hacia una búsqueda de incrementar su productividad y rentabilidad, sin olvidar el desarrollo sustentable de la región además de seguir aportando a la seguridad alimentaria (López, et al., 2017).

Lo anteriormente expuesto abre la pauta para describir el objetivo principal de esta investigación al elaborar y validar un instrumento que permita identificar las prácticas que las organizaciones agrícolas de la región de Guasave, Sinaloa, México han implementado para creación de valor garantizando su permanencia en el mercado y el cuidado el medio ambiente.

\section{MATERIALES Y MÉTODOS}

La población de estudio incurrió en los 32 dirigentes de organizaciones agrícolas del Municipio de Guasave, Sinaloa, México; registrados en el Directorio Estadístico Nacional de Unidades Económicas (DENUE) del Instituto Nacional de Estadística, Geografía e Informática (INEGI). Se aclara que, por ser una población 
pequeña, no se calculó ninguna muestra ya que fue posible recabar la información del total de la población estudio.

El enfoque que se retomó fue el cuantitativo no experimental, en su modalidad de exploratoria-descriptiva y correlacional, esto atendió las características de un fenómeno existente poco estudiado, como es la implementación de las prácticas sustentables y su impacto en la creación de valor por parte de las organizaciones estudiadas (Hernández, et al., 2010; Salkind, 1999).

El cuestionario fue aplicado en formato impreso, entregado de forma personal a los sujetos de estudio, aprovechando la ventaja que el contacto directo concede, permitiendo que se obtuvieran los resultados en un periodo corto de tiempo, aproximadamente 45 días.

\section{Análisis estadístico}

En cuanto al análisis de los datos que permiten revelar la fiabilidad y validez del instrumento, se utilizó el Paquete Estadístico para las Ciencias Sociales (Statistical Package for the Social Sciences, SPSS), versión 25 de IBM.

Para conocer la fiabilidad del cuestionario, el cual mide el grado en que la medida de una variable está libre de error aleatorio (Quero, 2010), se utilizó el Alpha de Cronbach, tomando como parámetros que, a mayor valor de Alfa, mayor fiabilidad. Los rangos de Alpha oscilan de 0 a 1 , donde, para las ciencias sociales, 0.80 se considera un valor aceptable (Bojórquez et al., 2013; Quero, 2010; y Celina y Campo, 2005). De igual forma, se obtuvieron las medidas descriptivas de media, varianza, y la prueba estadística de correlación de cada uno de los ítems.

Para medir la validez del instrumento se contó con la opinión de 10 expertos tanto académicos como profesionales, quienes evaluaron la pertinencia y claridad de los ítems que forman el instrumento, mediante una encuesta con una escala de Likert de 5 opciones, donde 1 es igual a "nada pertinente/claro"; 2: "poco pertinente/ claro"; 3: "normal, regular, ni bien ni mal"; 4 : "pertinente/claro" y 5: "muy pertinente/claro", en esta prueba se utilizaron la media y desviación estándar como pruebas estadísticas, con un nivel de significancia de $5 \%$, un nivel de confianza del $95 \%$ y un valor de decisión de Si $p \leq 0,05$ se rechaza la validez del instrumento.

\section{a) Proceso de diseño y construcción del cuestionario}

Este instrumento recabó la opinión de los dirigentes de las organizaciones agrícolas, sobre las diversas prácticas de sustentabilidad que llevan a cabo y que han funcionado para la creación de valor a sus organizaciones, el cual se aplicó a directores, gerentes y/o jefes de áreas.

La estructura del cuestionario se caracterizó por:

- Un bloque con variables descriptivas de identificación, "información general de la organización" que comprenden los ítems del 1 al 8 , que en su mayoría son preguntas abiertas.

- Dos bloques de contenido, el primero, con 23 ítems de escala valorativa, los cuales permiten recoger las prácticas de sustentabilidad llevadas a cabo en la organización; y el segundo, con la misma escala, recolecta información acerca del valor creado a partir de las prácticas sustentables, este con 17 ítems. Para ambos bloques se retomó la escala de Likert de 5 grados, que van de totalmente de acuerdo a totalmente en desacuerdo.

- Un ítem fue destinado para medir la rentabilidad a través de rangos alcanzados por las organizaciones durante los tres años. 
Como complemento a su estructura, fue necesario establecer dimensiones y subdimensiones de estudio, exclusivamente para los dos últimos bloques correspondientes a las variables de desarrollo sustentable y creación de valor respectivamente (ver Tabla 1), las cuales fueron útiles para identificar las prácticas de cada uno de los ejes que conforman la sustentabilidad y su impacto en la creación de valor tanto tangible como intangible.

Tabla 1. Constitución del instrumento: bloques, dimensiones, subdimensiones e ítems.

\begin{tabular}{|c|c|c|c|}
\hline Bloque & Dimensiones & Subdimensión & Ítems \\
\hline $\begin{array}{l}\text { 1. Información general } \\
\text { de la organización }\end{array}$ & & & $1-8$ \\
\hline \multirow{3}{*}{ 2. Desarrollo Sustentable } & Eje económico & & $9-14$ \\
\hline & Eje social & & $15-22$ \\
\hline & Eje ambiental & & $23-31$ \\
\hline \multirow{4}{*}{ 3. Creación de valor } & Tangibles & & $32-34$ \\
\hline & \multirow{3}{*}{ Intangibles } & $\begin{array}{l}\text { Imagen y reputación } \\
\text { de la organización }\end{array}$ & $35-37$ \\
\hline & & Cultura organizacional & $38-46$ \\
\hline & & Lealtad de los clientes & $47-49$ \\
\hline
\end{tabular}

Fuente: Autores.

\section{b) Prueba Piloto}

Con el fin de acercar el instrumento a la realidad, se procedió a realizar una prueba piloto a 5 colaboradores de organizaciones agrícolas de la región de Guasave, Sinaloa; seleccionadas a discreción del investigador del DENUE, tomando como base el conocimiento principal de los encuestados, esto con la finalidad de detectar posibles inconsistencias en el instrumento.

Esta prueba piloto permitió realizar la prueba estadística de Alpha de Cronbach, la cual permite conocer el grado de fiabilidad del instrumento, permitiendo en caso de salir con un valor bajo, hace las adecuaciones necesarias para que este aumente y sea aceptado, es decir, para que el instrumento sea considerado confiable.

\section{c) Revisión por expertos}

De un total de 10 expertos, se seleccionaron seis por su experiencia académica y el resto por su experiencia profesional y conocimiento práctico en el área de estudio, ambos grupos emitieron su juicio respecto a la pertinencia y claridad con la que se encuentran construidos cada uno de los ítems que conforman el cuestionario, esto mediante una escala de Likert de cinco opciones.

\section{RESULTADOS Y DISCUSIONES}

Este aporte surge de la necesidad de crear un instrumento que cuente tanto con un valor de 
fiabilidad y validez aceptable que recabe las opiniones relativas a las prácticas de sustentabilidad que contribuyan a la generación de valor dentro de las organizaciones agrícolas, con la predicción de ser aplicable en espacios con características similares. A continuación, se muestran los resultados que señalan su calidad en ambos aspectos:

\section{a) Análisis de fiabilidad}

Con el objetivo de determinar, en el instrumento original, y eliminar los ítems que generaban un impacto negativo se aplicó la prueba de fiabilidad de Alpha de Cronbrach (Tabla 2), el valor obtenido de la prueba piloto fue de 0.939 , lo que significa un valor elevado de fiabilidad.

Tabla 2. Prueba estadística Alpha de Cronbach en prueba piloto

\begin{tabular}{c|c|c}
\hline \multicolumn{2}{|c}{ Estadísticas de fiabilidad } \\
\hline Alfa de Cronbach & $\begin{array}{c}\text { Alfa de Cronbach basada en } \\
\text { elementos estandarizados }\end{array}$ & $\mathrm{N}$ de elementos \\
\hline 0,939 & 0,965 & 40 \\
\hline
\end{tabular}

Fuente: Autores.

Sin embargo, para poder aumentar la fiabilidad del instrumento, se analizó la correlación a través "escala si se elimina el elemento" del software utilizado, encontrando que los ítems 13, 25 y 45 arrojan un valor negativo (véase Tabla 3), por lo tanto, fue necesario eliminar el primero y último de estos debido a que se relacionaban con otros ítems y presentaron valores positivos. El ítem 25 se consideró debido a que dio información referente a energías renovables.

Debido a los objetivos que se buscan en los primeros ocho ítems, el de identificar el sujeto de estudio, estos no fueron considerados en la prueba piloto, de igual forma el número 34 debido a que tiene como objetivo analizar la rentabilidad del ente económico y sus posibles respuestas fueron de opción múltiple y no en la escala de Likert. (Ver anexo tabla 3).

Posteriormente, se hicieron las modificaciones señaladas, con el fin de incrementar el valor de Alfa, se procedió a aplicar el instrumento al total de las organizaciones agrícolas, y se volvió a realizar el análisis, obteniendo como resultado un valor de 0,941, es decir se aumentó la fiabilidad en dos milésimas y se erradicaron los ítems con valores negativos que sesgarían el instrumento.

\section{b) Análisis de Validez}

Salkind (1999) afirma que las investigaciones que utilicen como instrumento de recolección de datos un cuestionario estructurado es necesario que sea sometido a la prueba de validez, esto con el propósito de verificar que las variables midan lo que realmente se pretende medir. Retomando las diversas formas de validar el instrumento, se optó por validar su contenido, es decir, dar certeza a los ítems necesarios para poder dar respuesta los objetivos de la investigación (Delgado de Smith et al., 2002). Para hacer efectivo este tipo de validación es necesario que se haga a través del juicio de expertos (Soriano, 2014), con una gran experiencia profesional, académica y/o investigativa, relacionada con el tema de la investigación.

Para obtener los resultados de la validación del instrumento por expertos, fue necesario hacer uso herramientas de la estadística descriptiva, como la desviación estándar y la media. La Tabla 4 aprecia los resultados del análisis de validación por juicio de expertos. 
Tabla 4. Media y desviación estándar de la pertinencia y claridad de los ítems

\begin{tabular}{|c|c|c|c|c|}
\hline \multirow{2}{*}{ Dimensión } & \multirow{2}{*}{ Subdimensión } & \multirow{2}{*}{ Ítems } & \multicolumn{2}{|c|}{ Pertinencia y claridad } \\
\hline & & & Media & Desviación estándar \\
\hline \multirow{23}{*}{ Desarrollo Sustentable } & \multirow{6}{*}{ Eje Económico } & 9 & 4,10 & 0,876 \\
\hline & & 10 & 4,30 & 0,949 \\
\hline & & 11 & 4,50 & 0,972 \\
\hline & & 12 & 4,30 & 0,949 \\
\hline & & 13 & n.a & n.a \\
\hline & & 14 & 4,40 & 0,699 \\
\hline & \multirow{8}{*}{ Eje Social } & 15 & 4,40 & 0,699 \\
\hline & & 16 & 4,50 & 0,707 \\
\hline & & 17 & 4,30 & 0,675 \\
\hline & & 18 & 4,10 & 0,738 \\
\hline & & 19 & 4,70 & 0,483 \\
\hline & & 20 & 4,20 & 0,632 \\
\hline & & 21 & 4,30 & 0,675 \\
\hline & & 22 & 4,40 & 0,699 \\
\hline & \multirow{9}{*}{ Eje Ambiental } & 23 & 4,50 & 0,707 \\
\hline & & 24 & 4,60 & 0,966 \\
\hline & & 25 & 4,40 & 0,699 \\
\hline & & 26 & 4,30 & 0,949 \\
\hline & & 27 & 4,50 & 0,527 \\
\hline & & 28 & 4,60 & 0,516 \\
\hline & & 29 & 4,10 & 0,876 \\
\hline & & 30 & 4,70 & 0,675 \\
\hline & & 31 & 4,50 & 0,707 \\
\hline \multirow{18}{*}{ Creación de Valor } & \multirow{3}{*}{ Tangibles } & 32 & 4,30 & 0,949 \\
\hline & & 33 & 4,50 & 0,527 \\
\hline & & 34 & n.a & n.a \\
\hline & \multirow{15}{*}{ Intangibles } & 35 & 4,50 & 0,527 \\
\hline & & 36 & 4,30 & 0,675 \\
\hline & & 37 & 4,70 & 0,483 \\
\hline & & 38 & 4,50 & 0,527 \\
\hline & & 39 & 4,20 & 0,632 \\
\hline & & 40 & 4,10 & 0,738 \\
\hline & & 41 & 4,60 & 0,699 \\
\hline & & 42 & 4,40 & 0,516 \\
\hline & & 43 & 4,20 & 1.033 \\
\hline & & 44 & 4,70 & 0,483 \\
\hline & & 45 & n.a & n.a \\
\hline & & 46 & 4,30 & 0,675 \\
\hline & & 47 & 4,50 & 0,707 \\
\hline & & 48 & 4,40 & 0,699 \\
\hline & & 49 & 4,70 & 0,483 \\
\hline
\end{tabular}

Fuente: Autores. 
Con base en las valoraciones de los expertos, se puede ver que, en la dimensión de DS, en lo que respecta al eje económico, todos los ítems han sido evaluados por encima de la categoría de pertinentes y claros, con medias con valores superiores a los 4,10 puntos; en cuanto al eje social, las medias señalan que los ítems considerados son pertinentes y claros y muy pertinentes/claros, ya que su valor oscilo entre 4.10 y 4.70 ; en el eje ambiental, en su mayoría están considerados como muy pertinentes/claros, siendo el ítem 20 el que de acuerdo a las evaluaciones obtuvo la menor media con 4.10, entendiéndose que la forma de expresar lo que se quiere cuestionar no está muy clara, pero de acuerdo a la escala es considerado como pertinente/claro, que es una media considerada aceptable para este análisis.

Para la dimensión de creación de valor, en la subdimensión de tangibles, todos los ítems han sido valorados como pertinentes/claros y/o muy pertinentes/claros, con medias superiores a 4,30. Por último, los intangibles obtuvieron valores que van de lo pertinente y claro, con medias de 4,10 , a lo muy pertinente $y$ claro, cuyas medias ascienden a 4,70, siendo valores aceptados y significativamente positivos para la validación del instrumento.

En general, el instrumento cumple con los criterios de pertinencia y claridad (García-Garnica, 2016), sustentado por la evaluación de los expertos, ya que todos los ítems se encuentran por arriba de la media con valor de 4,1 que se considera como pertinente/claro; de los 38 ítems considerados en la evaluación hecha por los expertos, 21 fueron valorados como "pertinentes/claros" y el resto como "muy pertinentes/claros"; es decir, con esto se cumple la validación de contenido mediante la valoración por expertos.

\section{CONCLUSIONES}

El cuestionario "Prácticas sustentables para la creación de valor en organizaciones agrícolas" ha sido elaborado con el objetivo de recabar la opinión de los dirigentes de organizaciones agrícolas sobre las distintas prácticas de sustentabilidad que realizan y que han contribuido a la creación de valor de su empresa. El instrumento ha sido diseñado para ser atendido por mandos superiores de las organizaciones agrícolas, no se busca la opinión de los grupos de interés (Stakeholders) de esta actividad económica.

El instrumento garantiza y da respuesta al interrogante de que, con base en las prácticas responsables que estamos llevando como organización ¿estamos generando valor?, en cualquiera de sus variables. Se demostró que 32 empresas agrícolas, del municipio de Guasave, crean valor de forma tangible y son más rentables al tomar en cuenta el eje ambiental; es decir, aumentan sus utilidades y tienen cadenas de valor más sólidas. Sin embargo, se aclara, que los mismos entes económicos no han generado valor intangible a través de las prácticas que están realizado. Lo que permite concluir que, tras las diferentes pruebas realizadas, es posible, y se recomienda, la utilización de este instrumento para futuras investigaciones, replicándolo en otros contextos con características similares a las mostradas en las empresas agrícolas.

Este instrumento permite identificar las prácticas sustentables que contribuyen a la generación de valor en las organizaciones agrícolas, garantizándoles su permanencia en el mercado, ser más competitivas, mejorando su imagen y reputación, ser más rentables, pero, sobre todo, contribuyendo al logro del desarrollo sustentable del planeta. El instrumento no evalúa ni critica las practicas sustentables 
que realizan las organizaciones agrícolas, sino que diagnóstica el resultado de estas hacia la empresa en la generación de valor, es decir, si lo que están haciendo en el cuidado del medio ambiente y del entorno, les está dando dividendos tangibles e intangibles.

Considerando que de la sustentabilidad depende el futuro de los seres, las organizaciones agrícolas no pueden quedarse al margen de los cambios que se están experimentando a nivel mundial en relación con la urgente necesidad de equilibrar las tres dimensiones de la sustentabilidad. Enfocándose no solamente en la generación de riqueza, sino que sus actividades sean sostenibles y que las organizaciones, además de buscar la seguridad alimentaria, sean sensibles con respecto a los daños ecológicos generados por el impacto de sus actividades. La sustentabilidad debe constituir un eje rector para las instituciones y empresas para poder sobrevivir, generar valor y equilibrar las dimensiones económica, social y ambiental.

Es así como se aporta no solamente un instrumento validado, sino que también permitirá redefinir las líneas de acción y los compromisos institucionales en relación con la sustentabilidad, diseñando estrategias que favorezcan la generación de valor, tanto tangible como intangible, para que estas puedan permanecer y contribuyan a la sostenibilidad de los seres humanos en el planeta.

\section{CONTRIBUCIÓN DE LA AUTORÍA}

Primer autor: administración del proyecto, adquisición de recursos, análisis de datos, investigación y escritura - borrador original. Segundo autor: Investigación, análisis de datos, escritura - revisión y edición, logística y supervisión. Tercer autor: Metodología, conceptualización, revisión y edición. Cuarto autor: Conceptualización, metodología y supervisión.

\section{AGRADECIMIENTOS}

Al Consejo Nacional de Ciencia y Tecnología (Conacyt) por todos los apoyos otorgados para la realización de esa investigación; así como también a la Universidad Autónoma de Occidente por el aprendizaje brindado y apoyo de todos los investigadores que forman parte del núcleo básico de profesores del Doctorado en Sustentabilidad. A todos los empresarios agrícolas por su colaboración en el diseño de este instrumento, y finalmente a los docentes externos que contribuyeron en la validación de este.

\section{LITERATURA CITADA}

Bell, S., y Morse, S. (2018). Sustainable Indicators Past and Present. what next? Sustainability, 10 (5). https://doi.org/10.3390/su10051688

Bojórquez, J., López, L., Hernández, M., y Jiménez, E. (2013). Utilización del alfa de Cronbach para validar la confiabilidad de un instrumento de medición de satisfacción del estudiante en el uso del software Minitab. Eleventh LACCEI Latin American and Caribbean Conference for Engineering and Technology (LACCEI'2013)" Innovation in Engineering, Technology and Education for Competitiveness and Prosperity" August 14 - 16, 2013 Cancún, Mexico.

Carmona, E., y Magán, A. (2008). La estrategia ambiental: definición y tipologías. In University, Society and Global Markets. European Academic Publisehers, pp. 541-555.

Carrillo, G. (2011). Sustentabilidad en las Organizaciones: Nuevos enfoques y formas de entender del concepto. Administración y Organizaciones, 27 (14), 5-11.

Carro, J., Reyes, B., Rosano, G., Garnica, J., y Pérez, B. (2017). Modelo de Desarrollo Sustentable para la Industria de Recubrimientos Cerámicos. Revista Internacional de Contaminación Ambiental, 33 (1), 131-139. http://dx.doi.org/10.20937/ RICA.2017.33.01.12

Čater, T.; Prasnikar, J., y Čater, B. (2009). Environmental strategies and their motives and results in Slovenian business practice. Economic and Business Review, 11 (1), 55-74. 
Celina H. y Campo A., (2005). "Aproximación al uso del coeficiente alfa de Cronbach", Revista colombiana de psiquiatría, 34 (4), 572-580.

Consejo para el Desarrollo Económico de Sinaloa, CODESIN (2017). Reporte sobre la agricultura en Sinaloa al año 2016. Sinaloa en Numero 5. Recuperado de http://sinaloaennumeros.com/reporte-sobre-la-agricultura-en-sinaloa-al-ano-2016/ Consultado el día: 06 de enero de 2018

Corona, I. (2016). El desarrollo de la agricultura y el impacto que tendría en las finanzas públicas de México. Premio de las Finanzas Públicas 2016, México otorgado por el Centro de Estudios de las Finanzas Públicas (CEFP) de la Cámara de Diputados LXIII Legislatura. México.

Delgado de Smith, Y., Colombo L. y Rosmel, O. (2002). Conduciendo la Investigación. Editorial Comala. Caracas.

Elkington, J. (1994). Towards the Sustainable Corporation: Win-Win-Win Business Strategies for Sustainable Development. California Management Review, 36 (2), pp. 90-100. https://doi. org/10.2307/41165746.

Escobedo, G., y García, C. (2018). Responsabilidad Social Empresarial. En G. Escobedo, \& M. Andrade, Desarrollo Sustentable. Estrategias en las empresas para un futuro mejor (347-378). México: Alfaomega.

García-Garnica, M. (2016). Elaboración y validación de un cuestionario para medir prácticas eficaces de liderazgo pedagógico de la dirección. Revista de curriculum y profesorado, 20(3), 493-526.

Hart, S., y Milstein, M. (2003). Creating sustainable value. Academy of Management Executive, 17 (2), 56-69.

Henriques, A., y Richardson, J. (2013). The Triple Bottom Line, Does It All Add Up? Assessing the Sustainability of Business and CSR (en inglés). Earthscan.

Hernández, R., Fernández, C. y Baptista L. (2010). Metodología de la investigación. Quinta edición. México: Editorial Mac Graw Hill.

Jane, M. (2013). Environment Strategy. Universidad de Wonchester. Pp.1-6. Reino Unido.

Jaramillo, J.; Rodríguez, V.; Guzmán, M.; Zapata, M. y Rengifo, T. (2007). Manual técnico: Buenas
Prácticas Agrícolas en la Producción de tomate bajo condiciones protegidas. CORPOICA - MANA - Gobernación de Antioquia - FAO. Medellín, Colombia.

Leal, J. (2009). Desarrollo Sostenible en la empresa: Herramientas de Gestión. Presentación en Power Point el mes de mayo 2009. División de Desarrollo Sostenible y Asentamientos Humanos de la Comisión Económica para América Latina y El Caribe (CEPAL). Recuperado de https://www. cepal.org/ilpes/noticias/paginas/8/35988/joseLeal_DSEMPRESAHERRAMIENTAS.pdf

López, A., Calle, D., y Molina, A. (2017). Análisis del uso de herramientas de gestión ambiental en las empresas comerciales del Cantón de Morona. Killkana Sociales, 1(3), pp. 45-52. https://doi. org/10.26871/killkana_social.v1i3.62

Martínez, M.; Waliszewski, S.; Amador, O.; Meza, E.; Calderón, M.; Zenteno, E.; Huichapan, J.; Caba, M.; Félix, R. y Longoria, R. (2016). Aerial pesticide application causes DNA damage in pilots from Sinaloa, México. Environmental Science and PoIlution Research, 24 (3), 2412-2420. https://doi. org/10.1007/s11356-016-7974-5

Molina, E., y Victorero, E. (2015). La agricultura en países subdesarrollados. Particularidades de su financiamiento. Cuba: CLACSO.

IAEA (2012) Organismo Internacional de Energía Atómica. Agricultura y seguridad alimentaria: La contribución del OIEA. Departamento de cooperación técnica. Viena, Australia.

Orsato, R. (2006). Competitive Environmental Strategies: when does it pay to be green?. California Management Review, 48(2), 127-143. https://doi. org/10.2307/41166341

Paternoster, A. (2011) Herramientas para medir la sostenibilidad corporativa (Tesis de Maestría). Universidad Politécnica de Catalunia, Cataluña, España. En línea: https://upcommons.upc.edu/ bitstream/handle/2099.1/13644/PFM_Agustin Paternoster.pdf

Quero, M. (2010). Confiabilidad y coeficiente Alpha de Cronbach. Telos, 12 (2), 248-252.

Rodríguez, M. y Ricart, J. (2000). Estrategia medioambiental y creación de valor. Documento de investigación No.408. División de investigación de IESE. Universidad de Navarra. Barcelona, España. Bogotá, Colombia. 
Salkind, N. (1999). Métodos de investigación. México: Prentice Hall.

SAGARPA (2016) Secretaría de Agricultura, Ganadería, Desarrollo Rural, Pesca y Alimentación Cuarto informe de labores 2015-2016. pp. 151. México.

Sistema de Información Agroalimentaria y Pesquera (SIAP). (2018). Estadística de la producción agrícola del año 2015. Recuperado de https:// datos.gob.mx/busca/dataset/estadistica-de-laproduccion-agricola
Sinergia (2006). Producción Respetuosa en Viticultura. Impactos Ambientales en Agricultura. Proyecto Life Sinergia Life 03 ENV/E/000085 del Gobierno de La Rioja, España.

Soriano, A. (2014). Diseño y validación de instrumentos de medición. Diálogos, 8, 19-40.

Zulueta, A., Asencio, J., Leyva, D. y Montero. J. (2013). Sustentabilidad empresarial de proyectos mineros: el análisis multicriterio como perspectiva acertada para su evaluación. Minería y Geología, 29(4), 79-94. 